ISSN 0103-9954

\title{
QUALIDADE DE CHAPAS DE PARTÍCULAS DE Pinus elliottii COLADAS COM RESINA POLIURETANA SOB DIFERENTES COMBINAÇÕES DE PRESSÃO E TEMPERATURA
}

\author{
QUALITY OF Pinus elliottii PARTICLE BOARDS BONED WITH POLYURETHANE ADHESIVE \\ UNDER VARIOUS COMBINATIONS OF TEMPERATURE AND PRESSURE
}

\author{
Juarez Benigno Paes ${ }^{1}$ Shirley Tavares Nunes ${ }^{2}$ Francisco Antônio Rocco Lahr ${ }^{3}$ \\ Maria de Fátima Nascimento ${ }^{4}$ Roberta Maria de Albuquerque Lacerda ${ }^{5}$
}

\begin{abstract}
RESUMO
Os objetivos da pesquisa foram produzir chapas de partículas com resíduos do processamento da madeira de Pinus elliottii coladas com resina poliuretana à base de mamona e avaliar o efeito da combinação da pressão e temperatura na qualidade das chapas produzidas. Para tanto, foram confeccionadas 12 chapas, subdivididas em quatro tratamentos em função da pressão $(2,0 ; 3,0 ; 3,5$; e 3,5 MPa) e temperatura (90; 90; 50; e $60^{\circ} \mathrm{C}$ ). Depois de prensadas e climatizadas, as chapas foram seccionadas em corpos de prova, e realizados ensaios físico-mecânicos para determinação da densidade, inchamento e absorção de água (0-2h; 2-24h; e 0-24h); módulo de ruptura e de elasticidade em flexão estática, ligação interna e arrancamento de parafuso. As chapas prensadas com pressão de $3,0 \mathrm{MPa}$ e temperatura de $90^{\circ} \mathrm{C}$ e aquelas com pressão de $3,5 \mathrm{MPa}$ e temperatura de $60^{\circ} \mathrm{C}$ apresentaram os melhores resultados. A temperatura de prensagem foi a variável preponderante na qualidade das chapas produzidas.
\end{abstract}

Palavras-chave: aproveitamento de resíduo; madeira aglomerada; adesivo.

\begin{abstract}
This work aimed to produce homogeneous layer particle boards with residues of Pinus elliottii wood adhered with polyurethane adhesive to castor oil plant base and to evaluate the effect of the combination of pressure and temperature in the quality of the particle boards produced. To do so, 12 particle boards were manufactured, subdivided into four treatments in function of the pressure $(2.0 ; 3.0 ; 3.5$; and $3.5 \mathrm{MPa})$ and temperature $\left(90 ; 90 ; 50\right.$; and $\left.60^{\circ} \mathrm{C}\right)$. The particleboards were sectioned in test samples, and accomplished by physical-mechanical tests for the determination of density, swelling and absorption of water (0-2h; 2-24h; and 0-24h); rupture module and elasticity module in static bending, internal ligation and screw withdrawal. The particle boards pressed with pressure of $3.0 \mathrm{MPa}$ and temperature of $90{ }^{\circ} \mathrm{C}$ and that with pressure of $3.5 \mathrm{MPa}$ and temperature of $60^{\circ} \mathrm{C}$ presented the best results. The temperature of pressing was the predominant variable in the quality of those particle boards manufactured.
\end{abstract}

Keywords: residual use; particle board; adhesive.

1. Engenheiro Florestal, D.Sc., Professor do Departamento de Engenharia Florestal, Universidade Federal do Espírito Santo, Av. Governador Lindemberg, 316, Centro, CEP 29550-000, Jerônimo Monteiro (ES). jbp2@uol.com.br

2. Engenheira Florestal, Mestranda do Programa de Pós-Graduação em Ciências Florestais, Unidade Acadêmica de Engenharia Florestal, Universidade Federal de Campina Grande, Caixa Postal 64, CEP 58700-970, Patos (PB). shirlley_nunes@hotmail.com

3. Engenheiro Civil, Dr., Professor da Escola de Engenharia de São Carlos, Universidade de São Paulo, Laboratório de Madeira e Estrutura de Madeira, Av. Trabalhador Sãocarlense, 400, Centro, CEP 13566-590, São Carlos (SP). frocco@sc.usp.br

4. Arquiteta, Dra ${ }^{\mathrm{a}}$, Professora da Escola de Engenharia de São Carlos, Universidade de São Paulo, Laboratório de Madeira e Estrutura de Madeira, Av. Trabalhador Sãocarlense, 400, Centro, CEP 13566-590, São Carlos (SP). fati@sc.usp.br

5. Engenheira Florestal, Mestranda do Programa de Pós-Graduação em Ciências Florestais, Unidade Acadêmica de Engenharia Florestal, Universidade Federal de Campina Grande, Caixa Postal 64, CEP 58700-970, Patos (PB). robertaufcg@yahoo.com.br

Recebido para publicação em 8/07/2009 e aceito em 18/09/2010 


\section{INTRODUÇÃO}

Em função da disponibilidade $\mathrm{e}$ características físico-mecânicas, a madeira foi um dos primeiros materiais a ser utilizado pela humanidade e mesmo após o aparecimento de materiais sintéticos, ela continua a ser empregada como matéria-prima para inúmeros fins (JUVENAL e MATTOS, 2002). No entanto, a madeira é um material biológico altamente instável. Sua estrutura anatômica, bem como suas propriedades físicas, químicas e mecânicas variam entre espécies, e até mesmo dentro de uma mesma árvore (IWAKIRI et al., 2005).

A estrutura heterogênea da madeira, suas dimensões, a anisotropia e os defeitos naturais são fatores limitantes para uma série de utilizações. As dimensões das peças são limitadas pelo diâmetro e comprimento do tronco. O comportamento anisotrópico, que provoca variações das propriedades nas direções longitudinal, radial e tangencial e os defeitos como nós, inclinação de grãs, entre outros, são fatores que irão afetar diretamente o uso da madeira (MADEIRA TOTAL, 2008).

Em função das limitações existentes, e também visando um maior aproveitamento da madeira, iniciou-se a realização de pesquisas buscando alternativas para situações em que o uso da madeira sólida é inviável. Uma das opções foi o desenvolvimento de painéis à base de madeira, os quais possibilitam o aproveitamento de resíduos da indústria madeireira, fato que se encontra em constante evidência atualmente (MADEIRA TOTAL, 2008).

O melhor aproveitamento da matéria-prima tem sido a grande preocupação das indústrias de processamento mecânico da madeira. Nos países onde estas indústrias estão bem desenvolvidas, a utilização dos resíduos gerados durante $\mathrm{o}$ processamento é intensa, principalmente para a produção de celulose, chapas de partículas aglomeradas e energia (NOLASCO, 2000).

Assim, os painéis de madeira reconstituída a partir de resíduos podem ser considerados produtos ecologicamente corretos, pois utilizam materiais descartados e, portanto, contribuem para a diminuição do impacto da produção de bens de consumo proveniente dos recursos naturais (NASCIMENTO, 2003).

A quantidade de resíduos gerados pela indústria madeireira no Brasil é bastante elevada.
Apesar do volume representativo esses resíduos têm sido pouco utilizados e, quando isso ocorre, grande parte é destinada à produção de energia. Dentre esses resíduos, as maravalhas de madeira representam uma parcela significativa. As maravalhas apresentam boas características para a produção de chapas de partículas, permitindo a sua utilização, isolada ou em combinação com outras matérias-primas (BRITO, 1995). Atualmente utilizam-se, quase integralmente, os resíduos de processamento.

As chapas de partículas são produzidas com elementos de madeira, com a incorporação de adesivos, e consolidadas por meio da prensagem a quente. Seu processo de fabricação envolve a geração de partículas; secagem; classificação; aplicação do adesivo e aditivo químico; formação do colchão; pré-prensagem, prensagem a quente; acondicionamento; e acabamento (IWAKIRI, 1989).

Os adesivos de origem sintética são os mais utilizados atualmente, em função de suas melhores propriedades de adesão e ao ataque de micro-organismos, quando comparados aos adesivos de fontes naturais. Como os principais adesivos empregados nas indústrias utilizam o formaldeído, o qual quando liberado pode vir a acarretar problemas ao ambiente e a saúde dos seres humanos, muitos investimentos são feitos buscando um adesivo livre deste componente, mas que apresente a mesma eficácia (DIAS, 2008).

O emprego de adesivo poliuretano, obtido do óleo extraído da mamona, que é uma planta facilmente encontrada, ou cultivada, na maioria das regiões do Brasil, pode ser empregado na produção de chapas de madeira aglomerada, as quais são fabricadas atualmente com adesivos uréicos. Apesar do mercado promissor, o emprego da resina poliuretana derivada do óleo de mamona, desenvolvida pelo Instituto de Química de São Carlos da Universidade de São Paulo (SILVA, 2003) e fabricada pela KEHL Indústria e Comércio Ltda., sediada em São Carlos - SP, ainda não é empregada comercialmente pela indústria nacional de madeira aglomerada.

A competitividade do adesivo poliuretano em relação a outros polímeros existentes reside no fato de ser originário de matéria-prima natural e renovável, nos preços razoáveis dos di-isocianatos disponíveis no Brasil, e por não ser agressivo ao homem e ao ambiente (DIAS et al., 2005). Campos e Lahr (2007) realizaram testes a partir da utilização

Ci. Fl., v. 21, n. 3, jul.-set., 2011 
deste adesivo na fabricação de chapas de fibras de média densidade (MDF) de madeira de eucalipto, e verificaram suas interessantes características físico-mecânicas, além da viabilidade de variar os seus teores de acordo com a aplicação mais usual para cada uma das situações, conseguindo uma economia no consumo de adesivo.

O tempo e a temperatura de prensagem são parâmetros correlatos e são controlados para assegurar que a temperatura do miolo atinja o nível requerido para cura da resina. O tempo de prensagem deve ser também, o suficiente para possibilitar a migração do vapor d'água para as bordas da chapa e sua liberação até o término da prensagem (IWAKIRI, 1989).

A principal função da temperatura da prensa, na produção de painéis de partículas, é reduzir a resistência a compressão e facilitar a densificação do painel (MATOS, 1988). Iwakiri (1989) encontrou referências de que, quanto maior a temperatura de prensagem, maior será o fluxo de calor, permitindo melhor densificação das camadas mais internas dos painéis, resultando em maior resistência das ligações internas e melhor resistência à flexão estática. Dias (2008) obteve, para chapas produzidas com resíduos de Pinus e de Eucalyptus e resina poliuretana a base de mamona, resultados significativos para prensagem à temperatura de 60 e $90^{\circ} \mathrm{C}$.

A qualidade dos painéis é avaliada por meio de suas propriedades físico-mecânicas, tais como ligação interna; flexão estática; resistência ao arrancamento de pregos e parafusos; densidade; absorção de água; e inchamento em espessura (IWAKIRI et al., 2005).

Em função da quantidade de resíduos gerados pelas atividades agrícolas e florestais no Brasil e da necessidade de utilização dos resíduos para a confecção de novos materiais, esta pesquisa teve como objetivos produzir, em escala de laboratório, chapa de partículas com resíduos da madeira de Pinus elliottii Engelm, aderidos com adesivo poliuretano à base de mamona; e avaliar o efeito da combinação da pressão e temperatura na qualidade das chapas produzidas.

\section{MATERIAL E MÉTODOS}

\section{Local do experimento e matérias-prima utilizadas}

O experimento foi realizado no Laboratório de Madeiras e Estruturas de Madeiras (LaMEM) do Departamento de Estrutura de Madeira (DEM) da Escola de Engenharia de São Carlos (EESC) da Universidade de São Paulo (USP), Campus II, São Carlos, SP.

A matéria-prima utilizada na confecção das chapas foi proveniente de resíduos de processamento mecânico da madeira de Pinus elliottii Engelm, obtida por ocasião do processamento na plaina desempenadeira e desengrossadeira.

A resina utilizada foi à base de poliuretano derivada do óleo de mamona, que é uma resina do tipo bicomponente com tempo de gel de aproximadamente 25 minutos.

Nesta pesquisa, a resina comercial empregada foi composta por duas partes de poliól, utilizada como impermeabilizante e uma de isocianato aromático, como pré-polímero. É uma resina de cura a frio, que pode ser acelerada com temperatura de até $90^{\circ} \mathrm{C}$. A resina foi fornecida pela KEHL Indústria Química de São Carlos, sediada em São Carlos, SP.

\section{Obtenção das partículas e aplicação da resina}

Em função da necessidade de se utilizar material com granulometria mais homogênea, os resíduos foram quebrados em moinho, a fim de se obter partículas com 3 a $6 \mathrm{~mm}$ (comprimento e largura).

Para a confecção das chapas foram utilizadas 1.200 g de partículas, com $12 \%$ de umidade e $16 \%$ de adesivo líquido em relação à massa de partículas secas. Esta quantidade foi dividida de forma que a proporção de adesivo e catalisador fosse de 2:1, respectivamente.

As partículas, o adesivo e o catalisador, em quantidade pré-estabelecida para cada chapa, foram pesados em uma balança com precisão de 0,01g. $\mathrm{O}$ adesivo e o catalisador foram misturados, homogeneizados e adicionados às partículas.

As partículas foram misturadas ao adesivo de forma manual durante aproximadamente 5 minutos e posteriormente em uma misturadora por mais 5 minutos.

\section{Formação e pré-prensagem do colchão}

As partículas de madeira com o adesivo foram transferidas para uma caixa formadora, confeccionada de madeira, com dimensões laterais de 40 × $40 \mathrm{~cm}$, e sob a mesma foi colocada uma placa de aço de $3 \mathrm{~mm}$ de espessura. Para evitar que as partículas aderissem à placa, sobre a mesma foi posta uma folha de papel alumínio. 
O material foi pré-prensado manualmente para acomodação das partículas e a formadora foi removida e adicionada outra placa e folha de papel alumínio sobre o colchão formado e dispostos dois espaçadores de $10 \mathrm{~mm}$, entre as placa de aço, para assegurar a espessura final da chapa durante a prensagem, para produzir chapas com densidade nominal de $740 \mathrm{~kg} / \mathrm{m}^{3}$.

\section{Prensagem das chapas}

A prensagem a quente das chapas foi em uma prensa hidráulica Marconi MA 098/50, que tem capacidade de $500 \mathrm{kN}$, temperatura máxima de $200^{\circ} \mathrm{C}$ para fabricar chapas em escala de laboratório, com pressão em torno de $5 \mathrm{MPa}$. O tempo de prensagem foi de 10 minutos com a variação da temperatura e pressão conforme apresentado na Tabela 1. Para cada combinação (pressão e temperatura de prensagem) foram confeccionadas três chapas.

\section{Aclimatização, esquadrejamento e confecção dos corpos de prova}

Depois de prensadas, as chapas foram identificadas, conforme tratamento (Tabela 1), climatizadas por dois dias e esquadrejadas, ficando com as dimensões finais de $35 \times 35 \mathrm{~cm}$.

Os corpos de prova foram confeccionados na oficina do LaMEM, conforme preconizado pela Norma Brasileira Regulamentadora - NBR 14810-3 da Associação Brasileira de Normas Técnicas (ABNT, 2002a).

Para a determinação das propriedades físicomecânicas foram utilizados corpos de prova com as seguintes dimensões: densidade $(50$ x $50 \mathrm{~mm})$; inchamento e absorção de água ( 25 x $25 \mathrm{~mm})$, sendo empregados 10 corpos de prova por tratamento, retirados aleatoriamente das três chapas de cada combinação; flexão estática $(50$ x $250 \mathrm{~mm})$; tração perpendicular às fibras ou ligação interna $(50$ x 50 $\mathrm{mm})$; arrancamento de parafusos ( $75 \times 150 \mathrm{~mm})$, com o emprego de sete corpos de prova de cada chapa produzida, totalizando 21 corpos de prova.

TABELA 1: Pressões e temperaturas utilizadas na confecção das chapas aglomeradas.

TABLE 1: Pressures and temperatures used in the particleboards manufacture.

\begin{tabular}{ccccc}
\hline \multirow{2}{*}{ Combinações } & \multicolumn{4}{c}{ Tratamentos } \\
\cline { 2 - 5 } & 1 & 2 & 3 & 4 \\
\hline Pressão $(\mathrm{MPa})$ & 2,0 & 3,0 & 3,5 & 3,5 \\
Temperatura $\left({ }^{\circ} \mathrm{C}\right)$ & 90 & 90 & 50 & 60 \\
\hline
\end{tabular}

\section{Ensaios físico-mecânicos dos corpos de prova}

Antes da realização dos ensaios foram determinadas as dimensões reais (largura, espessura e comprimento) de cada corpo de prova, com o uso de paquímetro digital com precisão de $0.01 \mathrm{~mm}$.

Para a determinação das propriedades mecânicas das chapas foi utilizada uma máquina universal de ensaio da marca Dartec, com capacidade de 10 toneladas $(100 \mathrm{kN})$.

\section{Densidade das chapas e inchamento em espessura e absorção de água das chapas}

$\mathrm{Na}$ determinação da densidade, mediram-se a espessura em cinco pontos do corpo de prova (sendo uma no centro e as demais nas bordas), o comprimento e a largura com o emprego de paquímetro com precisão de $0,01 \mathrm{~mm}$ e a massa do corpo de prova em balança com precisão de $0,01 \mathrm{~g}$.

Para o inchamento, mediram-se a espessura do corpo de prova, com precisão de $0,01 \mathrm{~mm}$, e a massa com precisão de $0,01 \mathrm{~g}$. Após as medições, os corpos de prova foram submersos um recipiente com água destilada.

Depois de transcorridas 2 e 24 horas de imersão, os corpos de prova foram retirados do recipiente, secos com papel absorvente e, novamente medidos, tendo e o inchamento e absorção de água sido determinados.

\section{Flexão estática, tração perpendicular ou ligação interna e arrancamento de parafusos \\ No ensaio de flexão estática foram} determinados os módulos de elasticidade (MOE) e de ruptura (MOR) ao ser adotada uma velocidade de ensaio de $6 \mathrm{~mm} / \mathrm{min}$.

Para a realização dos ensaios de tração perpendicular, lixaram-se as faces do corpo de prova e mediram-se a largura e o comprimento com precisão de $0,01 \mathrm{~mm}$. Os corpos de prova foram colados nos blocos de tração, conforme recomendado pela ABNT (2002a). Depois da colagem, o conjunto formado por corpo de prova e bloco de tração foi acondicionado em condições de laboratório $( \pm 12 \%$ de umidade de equilíbrio) e o ensaio foi realizado ao adotar uma velocidade de ensaio de $4 \mathrm{~mm} / \mathrm{min}$.

Para o ensaio de arrancamento de parafuso, produziu-se, com o auxílio de uma broca de $2,8 \mathrm{~mm}$ de diâmetro, um orifício com profundidade de 7 $\mathrm{mm}$ no centro da lateral do corpo de prova e fixouse um parafuso autorrosqueável, e empregou-se uma velocidade de ensaio de $15 \mathrm{~mm} / \mathrm{min}$. Os resultados foram registrados em Newtons, com exatidão de $0,1 \mathrm{~N}$.

Ci. Fl., v. 21, n. 3, jul.-set., 2011 


\section{Análise estatística empregada}

Para a análise e avaliação dos resultados foi utilizado um delineamento inteiramente casualisado em que foram comparadas as propriedades físicomecânicas das chapas produzidas. Para avaliar as propriedades mecânicas, foram retiradas sete amostras de cada chapa confeccionada, totalizando 21 amostras. Para a análise das propriedades físicas, foram empregadas 10 amostras retiradas aleatoriamente das chapas produzidas para cada combinação testada (tratamento).

Os valores de inchamento e de absorção de água, por se tratarem de dados em porcentagem, quando necessários, para permitir a análise de variância, foram transformados em arcsen (raiz quadrada (valor em porcentagem/100)), como o recomendado por Steel e Torrie (1980).

$\mathrm{Na}$ análise e avaliação dos ensaios foi empregado o teste de Tukey a $5 \%$ de significância, para comparar as médias dos tratamentos detectados como significativos pelo teste de F.

\section{RESULTADOS E DISCUSSÃO}

São apresentados e discutidos os resultados dos ensaios realizados para a determinação das propriedades físicas e mecânicas das chapas confeccionadas com partículas de Pinus elliottii aderidas com resina poliuretana à base de mamona.

\section{Propriedades físicas das chapas}

Os resultados dos testes de densidade, absorção de água e inchamento em espessura (após imersão em água por 2 e 24 horas) estão apresentados na Tabela 2 .

Os valores médios de densidade, obtidos no ensaio realizado, foram de aproximadamente $700 \mathrm{~kg} / \mathrm{m}^{3}$ para as chapas dos tratamentos 1 e 3 , e superiores a $800 \mathrm{~kg} / \mathrm{m}^{3}$ para as dos tratamentos $2 \mathrm{e}$ 4.

As condições de pressão e temperatura adotadas nos tratamentos 2 e 4 causaram uma melhor compactação do colchão, aumentando a densidade das chapas. Para as mesmas condições de pressão (tratamentos 3 e 4), a maior temperatura produziu chapas de maior densidade. Esses valores indicam que a temperatura de prensagem foi uma das variáveis preponderantes no incremento da densidade das chapas produzidas.

Os valores de densidade obtidos estão em conformidade com a norma comercial americana, "Commercial Standard - CS 236/66" (1968) para chapas de partículas de madeira de média e alta densidade.

Dos dados de inchamento apresentados na Tabela 2, apenas aquele do tratamento 2 atingiu o valor estabelecido pela NBR 14810 da ABNT (2002b) que é de no máximo $8 \%$ para o inchamento dos aglomerados após 2 horas de imersão em água. Os demais tratamentos apresentaram valores médios, de inchamento em 2 horas, superiores ao exigido pela norma. Estes resultados estão em conformidade com os valores encontrados por Dias et al. (2008) para chapas produzidas com resíduos de Pinus sp. aderidos com resina poliuretana à base de mamona, que variaram na faixa de 9 a $14 \%$.

Como a quantidade de partículas e de adesivo empregada, a espessura das chapas confeccionadas e o tempo de prensagem foram semelhantes, as chapas confeccionadas sob maiores pressões e temperaturas (tratamentos 2 e 4) apresentaram menor tendência a incharem durante a climatização.

Exceto para o tratamento 2, o maior inchamento das chapas ocorreu durante as duas primeiras horas de imersão, com um menor inchamento entre 2 e 24 horas. Após 24

TABELA 2: Valores médios de densidade $\left(\mathrm{kg} / \mathrm{m}^{3}\right)$, absorção de água $(\%)$ e inchamento em espessura das chapas $(\%)$.

TABLE 2: Medium values of density $\left(\mathrm{kg} / \mathrm{m}^{3}\right)$, water absorption $(\%)$ and swell in thickness of particleboards $(\%)$.

\begin{tabular}{|c|c|c|c|c|c|c|c|c|}
\hline \multicolumn{2}{|c|}{ Tratamentos } & \multirow{2}{*}{$\begin{array}{c}\text { Densidade } \\
\left(\mathrm{kg} / \mathrm{m}^{3}\right)\end{array}$} & \multicolumn{3}{|c|}{ Inchamento em espessura $(\%)$} & \multicolumn{3}{|c|}{ Absorção de água (\%) } \\
\hline $\begin{array}{c}\text { Pressão } \\
\text { (MPa) }\end{array}$ & $\begin{array}{c}\text { Temperatura } \\
\left({ }^{\circ} \mathrm{C}\right)\end{array}$ & & $0-2 h$ & $2-24 h$ & $0-24 h$ & $0-2 h$ & $2-24 h$ & $0-24 h$ \\
\hline $1-2,0$ & 90 & $720 \mathrm{~b}$ & $9,71 \mathrm{ab}$ & $6,59 \mathrm{~b}$ & $16,91 \mathrm{a}$ & $20,73 \mathrm{a}$ & $18,97 \mathrm{a}$ & $43,70 \mathrm{a}$ \\
\hline $2-3,0$ & 90 & $890 \mathrm{a}$ & $7,43 \mathrm{~b}$ & $8,19 \mathrm{a}$ & $15,51 \mathrm{a}$ & $3,91 \mathrm{c}$ & $12,16 \mathrm{~b}$ & $16,54 \mathrm{c}$ \\
\hline $3-3,5$ & 50 & $700 \mathrm{~b}$ & $11,04 \mathrm{ab}$ & $5,97 \mathrm{c}$ & $17,65 \mathrm{a}$ & $19,62 \mathrm{a}$ & $17,58 \mathrm{a}$ & $40,63 \mathrm{ab}$ \\
\hline $4-3,5$ & 60 & $840 \mathrm{a}$ & $14,09 \mathrm{a}$ & $7,65 \mathrm{a}$ & $21,88 \mathrm{a}$ & $10,59 \mathrm{~b}$ & $18,49 \mathrm{a}$ & $31,09 \mathrm{~b}$ \\
\hline
\end{tabular}

As médias seguidas por uma mesma letra, na vertical, em cada seção, não diferem entre si (Tukey; $p>0,05$ ). 
horas de imersão não foi observada diferença significativa entre o inchamento das chapas. Porém, numericamente, as chapas dos tratamentos 1 e 2 incharam menos. Isto indica que a temperatura de prensagem foi uma variável preponderante na cura do adesivo utilizado.

A NBR 14810 da ABNT (2002b) não estabelece valores de inchamento em 24 horas para as chapas aglomeradas, mas, os valores obtidos (Tabela 2) foram inferiores aos encontrados por Dias (2008), para chapas produzidas com resíduos de Pinus sp. e Eucalyptus sp. aderidos com resina poliuretana à base de mamona.

A norma CS 236/66 (1968) estabelece, para chapas de partículas de madeira de média e alta densidade, valores de 35 e 55\% para o inchamento em espessura. De acordo com essa norma, os valores obtidos para as chapas produzidas na presente pesquisa (Tabela 2) estão em conformidade com tais referências.

A NBR 14810 (ABNT, 2002b) não apresenta exigências quanto à absorção de água dos aglomerados. No entanto, esta propriedade foi avaliada tendo os valores obtidos nos tratamentos 2 e 4 sido inferiores aos obtidos por Dias (2008), que variaram de 12,30 a $14,35 \%$, após duas horas de imersão. Segundo Kelly (1977), a absorção de água aumenta com a diminuição na densidade dos painéis, tendo o autor encontrado resultados que variaram de 28,27 a $36,21 \%$ para absorção em 24 horas, que foram inferiores aos obtidos para as chapas de maior densidade (Tabela 2).

As chapas produzidas com pressão de 3,0 $\mathrm{MPa}$ e temperatura de prensagem de $90^{\circ} \mathrm{C}$ absorveram menos água que as demais, indicando que estas condições de confecção foram as melhores dentre as situações avaliadas.

\section{Propriedades mecânicas das chapas \\ Ensaios de flexão estática}

Os ensaios de resistência na flexão estática apresentaram diferenças estatísticas entre os módulos de ruptura (MOR) e de elasticidade (MOE) conforme a variação de temperatura e pressão de prensagem das chapas (Tabela 3 ).

As chapas produzidas com a pressão de $3,0 \mathrm{MPa}$ e temperatura de $90^{\circ} \mathrm{C}$ (tratamento 2) apresentaram maiores valores de resistência (MOR) e de rigidez (MOE) que as demais e aquelas produzidas com a pressão de 3,5 MPa e temperatura de $50^{\circ} \mathrm{C}$ os menores valores. Como as chapas dos tratamentos 2, 3 e 4 (Tabela 2) foram submetidas a pressões de prensagem semelhantes ou muito próximas, os resultados indicam que a temperatura de prensagem foi a variável preponderante na qualidade das chapas para os quesitos resistência e rigidez na flexão estática dos painéis produzidos.

Os valores de resistência apresentados pelas chapas produzidas não alcançaram o mínimo de $18 \mathrm{MPa}$ estabelecido pela NBR 14810 da ABNT (2002b) para chapas de aglomerado com espessura compreendida entre 8 e $13 \mathrm{~mm}$, tampouco o estabelecido pela norma comercial americana CS 236-66 (1968) para painéis de alta ou média densidade, com relação à resistência e à rigidez dos painéis.

Dias et al., (2008) também constataram este problema, e relatam que os baixos valores de MOE obtidos podem ter ocorrido em função da má distribuição do adesivo, durante o processo de formação dos painéis.

\section{Tração perpendicular às fibras e arrancamento de parafusos}

$\mathrm{O}$ ensaio de tração perpendicular às fibras (ligação interna) (Tabela 4) indicou que os painéis produzidos sob pressão de 3,0 $\mathrm{MPa}$ e temperatura de $90^{\circ} \mathrm{C}$ (tratamento 2) apresentaram maior valor de ligação interna, sendo este estatisticamente igual aos painéis confeccionados com o emprego de 2,0 MPa e temperatura de $90^{\circ} \mathrm{C}$ (tratamento 1). Como os painéis do tratamento 4 (pressão de 3,5 MPa e temperatura de $60^{\circ} \mathrm{C}$ ) apresentaram valores inferiores ao do tratamento 2 , a temperatura de prensagem foi a variável que contribuiu para a melhor característica dos painéis.

A NBR 14810 (ABNT, 2002b) recomenda valores de 0,40 MPa para tração perpendicular às fibras (ligação interna) de painéis aglomerados com espessura de 8 a $13 \mathrm{~mm}$. Já a norma americana CS 236/66 (1968), valores de 0,49 e 1,40 MPa para painéis aglomerados de média e alta densidade, respectivamente. Todas as condições de temperatura e pressão analisadas apresentaram valores de tração perpendicular às fibras superiores às exigências das normas brasileiras e americanas (Tabela 4). Destacase, que alguns pesquisadores, dentre eles Matos (1988), Brito (1995), Nascimento (2003), Iwakiri et al. (2005), Dias et al. (2005; 2008), Dias (2008), consideram este ensaio como o mais importante para definir a qualidade de painéis aglomerados.

Os valores médios de arrancamento de parafusos para os tratamentos 1,2 e 4 foram semelhantes estatisticamente, tendo o tratamento 3 
TABELA 3: Valores médios dos testes de flexão estática (MOR e MOE).

TABLE 3: Medium values of static bending tests (MOR and MOE).

\begin{tabular}{cccc}
\hline \multicolumn{2}{c}{ Tratamentos } & Modulo de ruptura & $\begin{array}{c}\text { Modulo de elasticidade } \\
(\mathrm{MPa})\end{array}$ \\
\cline { 1 - 3 } $\begin{array}{c}\text { Pressão } \\
(\mathrm{MPa})\end{array}$ & $\begin{array}{c}\text { Temperatura } \\
\left({ }^{\circ} \mathrm{C}\right)\end{array}$ & & \\
\hline $1-2,0$ & 90 & $8,76 \mathrm{c}$ & $1065,70 \mathrm{c}$ \\
$2-3,0$ & 90 & $14,42 \mathrm{a}$ & $1636,38 \mathrm{a}$ \\
$3-3,5$ & 50 & $5,73 \mathrm{~d}$ & $675,29 \mathrm{~d}$ \\
$4-3,5$ & 60 & $11,43 \mathrm{~b}$ & $1364,23 \mathrm{~b}$ \\
\hline
\end{tabular}

As médias seguidas por uma mesma letra, na vertical, em cada seção, não diferem entre si (Tukey; $p>0,05$ ).

TABELA 4: Valores médios dos ensaios de ligação interna (MPa) e arrancamento de parafuso (N).

TABLE 4: Medium values of internal ligament trials (MPa) and screw withdrawal (N).

\begin{tabular}{|c|c|c|c|}
\hline \multicolumn{2}{|c|}{ Tratamentos } & \multirow[b]{2}{*}{$\begin{array}{l}\text { Ligação interna } \\
\text { (Mpa) }\end{array}$} & \multirow[b]{2}{*}{$\begin{array}{l}\text { Arrancamento de parafusos } \\
\qquad(\mathrm{N})\end{array}$} \\
\hline $\begin{array}{l}\text { Pressão } \\
(\mathrm{MPa})\end{array}$ & $\begin{array}{l}\text { Temperatura } \\
\left({ }^{\circ} \mathrm{C}\right)\end{array}$ & & \\
\hline $1-2,0$ & 90 & $1,41 \mathrm{ab}$ & $5,58 \mathrm{ab}$ \\
\hline $2-3,0$ & 90 & $1,72 \mathrm{a}$ & $6,29 \mathrm{a}$ \\
\hline $3-3,5$ & 50 & $0,85 \mathrm{c}$ & $4,62 \mathrm{~b}$ \\
\hline $4-3,5$ & 60 & $1,36 \mathrm{~b}$ & $6,14 \mathrm{a}$ \\
\hline
\end{tabular}

As médias seguidas por uma mesma letra, na vertical, em cada seção, não diferem entre si (Tukey; p > 0,05).

apresentado resultado semelhante ao tratamento 1 e inferior aos tratamentos 2 e 4 (Tabela 4). A NBR 14810 (ABNT, 2002b) indica que este ensaio não é aplicável para chapas de espessura de 8 a $13 \mathrm{~mm}$.

\section{CONCLUSÕES}

As chapas confeccionadas com pressões de 3,0 e 3,5 MPa e temperatura de prensagem de 90 e $60{ }^{\circ} \mathrm{C}$ apresentaram altas densidades e valores médios de inchamento em 2 horas e de absorção de água em conformidade com a norma brasileira e comercial americana.

O emprego da pressão de 3,0 $\mathrm{MPa}$ e temperatura de prensagem de $90^{\circ} \mathrm{C}$ proporcionaram melhor qualidade às chapas confeccionadas.

Dentre as variáveis testadas, a temperatura foi preponderante na qualidade das chapas produzidas, tendo as chapas confeccionadas com a temperatura de 60 e $90{ }^{\circ} \mathrm{C}$, apresentado melhor resistência, rigidez e ligação interna que as chapas prensadas com pressão de 3,5 MPa e temperatura de prensagem de $50^{\circ} \mathrm{C}$.

Independente das condições de pressão e temperatura utilizadas, as chapas produzidas apresentaram valores de tração perpendicular às fibras (ligação interna) superiores às exigências da norma brasileira e da comercial americana.
Em função da qualidade das chapas produzidas o emprego da resina poliuretana à base de óleo de mamona é tecnicamente viável na fabricação de painéis aglomerados.

\section{REFERÊNCIAS BIBLIOGRÁFICAS}

ASSOCIAÇÃO BRASILEIRA DE NORMAS TÉCNICAS - ABNT - NBR 14810-2. Chapas de madeira aglomerada. Parte 2 - Requisitos. Rio de Janeiro: ABNT, 2002b. 4 p.

ASSOCIAÇÃO BRASILEIRA DE NORMAS TÉCNICAS - ABNT - NBR 14810-2. Chapas de madeira aglomerada. Parte 3 - Métodos de ensaio. Rio de Janeiro: ABNT, 2002a. 32 p.

BRITO, E.O. Produção de chapas de partículas de madeira a partir de maravalhas de Pinus elliottii plantado no sul do Brasil. 1995. 123 f. Tese (Doutorado em Ciências Florestais) - Universidade Federal do Paraná, Curitiba, 1995.

CAMPOS, C. I.; LAHR, F. A. R. Pinus e eucalipto para produção de painéis. Revista da Madeira. Curitiba, v. 21, p. 229 - 232, 2007.

COMMERCIAL STANDARD - CS 236-66. Mat formed wood particleboard. [S.1.], 1968.

DIAS, F. M. Aplicação de resina poliuretana à base de mamona na fabricação de painéis de madeira aglomerada. In: LAHR, F. A. R. Produtos 
derivados da madeira. São Carlos: EESC/USP, 2008. p. 37-160.

DIAS, N. A. B. et al. Aproveitamento de resíduos de Pinus sp. para a produção de chapas de partículas utilizando resina poliuretana bicomponente à base de óleo de mamona. São Carlos: LaMEM, EESC, USP, 2008.12 p.

DIAS, F. M. et al. Relation between the compaction rate and physical and mechanical properties of particleboards. Materials Research, São Carlos, v. 8, n. 3, p. 329-333, 2005.

IWAKIRI, S. A influência de variáveis de processamento sobre propriedades de chapas de partículas de diferentes espécies de Pinus. 1989. 130 f. Tese (Doutorado em Ciências Florestais) Universidade Federal do Paraná, Curitiba, 1989.

IWAKIRI, S. et al. Painéis de madeira aglomerada. In: IWAKIRI, S. (Ed.). Painéis de madeira reconstituída. Curitiba: FUPEF, 2005. p.123 - 166. JUVENAL, T. L.; MATTOS, L. L. G. O setor florestal no Brasil e a importância do reflorestamento. BNDES Setorial, Rio de Janeiro, n. 16, p. 3 - 30, 2002.

KELLY, M. W. Critical literature review of relationship between processing parameters and physical properties of particleboard. Madison: USDA Forest Service, 1977. 66 p. (FPL Report, 10).

MADEIRA TOTAL. Estrutura físico-química da madeira. Disponível em: <http://www. madeiratotal.com.br/ntc.asp?Cod $=2980>$. Acesso em: 12 de novembro de 2008.

MATOS, J. L. M. Ciclo de prensa em chapas de partículas estruturais "Waferboards". 1988. 163 f. Dissertação (Mestrado em Ciências Florestais) Universidade Federal do Paraná, Curitiba, 1988.

NASCIMENTO, M. F. Chapas de partículas homogêneas: madeiras do nordeste do Brasil. 2003. 143 f. Tese (Doutorado em Engenharia de Materiais)-Escola de Engenharia de São Carlos, Universidade de São Paulo, São Carlos2003.

NOLASCO, A. M. Resíduos da colheita e beneficiamento da caixeta - Tabebuia cassinoides (Lam.) DC.: caracterização e perspectivas. 2000. 212 f. Tese (Doutorado em Ciências da Engenharia Ambiental)-Escola de Engenharia de São Carlos, Universidade de São Carlos, São Carlos, 2000.

SILVA, S. A. M. Chapa de média densidade (MDF) fabricada com poliuretana mono-componente derivada de óleo de mamona: caracterização por método destrutivo e ultrasom. 2003. $234 \mathrm{f}$. (Doutorado em Engenharia Agrícola)-Faculdade de Engenharia Agrícola, Universidade Estadual de Campinas, Campinas, 2003.

STEEL, R. G. D.; TORRIE, J. H. Principles and procedures of statistic: a biometrical approach. 2nd ed. New York: Mc-Graw Hill, 1980. 633 p. 